\title{
Application of independent component analysis to ac dipole based optics measurement and correction at the Relativistic Heavy Ion Collider
}

\author{
X. Shen* and S. Y. Lee \\ Department of Physics, Indiana University, Bloomington, Indiana 47405, USA \\ M. Bai, S. White, G. Robert-Demolaize, Y. Luo, and A. Marusic \\ Brookhaven National Laboratory, Upton, New York 11973, USA \\ R. Tomás \\ CERN, CH1211 Geneva 23, Switzerland \\ (Received 23 July 2013; published 12 November 2013)
}

\begin{abstract}
Correction of beta-beat is of great importance for performance improvement of high energy accelerators, like the Relativistic Hadron Ion Collider (RHIC). At RHIC, using the independent component analysis method, linear optical functions are extracted from the turn by turn beam position data of the ac dipole driven betatron oscillation. Despite the constraint of a limited number of available quadrupole correctors at RHIC, a global beta-beat correction scheme using a beta-beat response matrix method was developed and experimentally demonstrated. In both rings, a factor of 2 or better reduction of beta-beat was achieved within available beam time. At the same time, a new scheme of using horizontal closed orbit bump at sextupoles to correct beta-beat in the arcs was demonstrated in the Yellow ring of RHIC at beam energy of $255 \mathrm{GeV}$, and a peak beta-beat of approximately $7 \%$ was achieved.
\end{abstract}

PACS numbers: 41.75.-i, 41.85.- p, 29.27.-a

\section{INTRODUCTION}

There have been many proposals for improving performance of the Relativistic Hadron Ion Collider (RHIC), such as electron lenses for head-on beam-beam compensation, beta star knob at interaction region for dynamic beta squeezing, near-integer working point for larger dynamical aperture, as well as more tune space for preserving polarization [1]. All of these projects and proposals require a solid understanding as well as precise control of machine optics. Accurate optics measurement techniques and efficient optics correction schemes are therefore desired to minimize the deviation of measured beta functions away from designed beta functions, i.e., beta-beat.

For optics measurements, RHIC is equipped with both pulsed kickers and ac dipoles [2] to excite coherent beam motion. A pulsed kicker can excite coherent free betatron oscillation. However, the excited free oscillation is often prone to quick decoherence which compromises the quality of the signal. In addition, the excitation can cause beam emittance growth. On the other hand, an ac dipole operated in an adiabatic fashion is able to excite a sustained coherent driven betatron oscillation of the beam with a large amplitude and preservation of beam emittance [3]. Higher signal

\footnotetext{
*xiaoshen@indiana.edu

Published by the American Physical Society under the terms of the Creative Commons Attribution 3.0 License. Further distribution of this work must maintain attribution to the author(s) and the published article's title, journal citation, and DOI.
}

to noise ratio as well as nondestructive nature makes ac dipole a preferable diagnostic tool for high energy hadron accelerators.

The turn by turn data from a linear beam position monitor (BPM) system can be considered as a linear mixture of physical source signals, which are usually harmonic oscillations with different frequencies, such as betatron motion, synchrotron motion, and nonlinear motions. These source signals are closely related to the machine optics. Many methods have been developed to derive linear optics from turn by turn BPM data by separating the source signals. For example, a Fourier analysis technique was implemented at PEP-II to extract four linear independent betatron orbits from turn by turn BPM data for linear optics modeling [4]. The technique of principle component analysis (PCA) was also applied at the Advanced Photon Source (APS) for linear optics measurement [5]. Independent component analysis (ICA) is a powerful technique in signal processing, and particularly efficient in separating narrowband source signals from sampled data [6]. Both spatial and temporal functions of different source signals extracted from ICA analysis provide information about beam motions from which optical functions can be derived. In addition to its high efficiency in mode separation, the technique of ICA for optics measurement has been proven to be as robust as PCA against BPM noise [7]. ICA has been applied to the free betatron oscillation signal to analyze the transverse betatron amplitude function and phase advance, dispersion function, linear coupling, and sextupole strength [7-9]. However, additional considerations on interpretation of source signals are required to apply ICA to ac dipole driven 
betatron oscillation for optics measurement. This is because the measured optics are modified in driven beam oscillations excited by an ac dipole [10].

There are various methods to correct beta-beat, such as stop band compensation [11] and linear optics from closed orbit [12] technique. The stop band compensation method relies on the fact that beta-beat is dominated by harmonics near twice the betatron tune. The harmonic contents of the measured beta-beat can be obtained by Fourier analysis. A few families of trim quadrupoles are then excited to cancel the dominating harmonics [13]. Since the horizontal and vertical betatron tunes for RHIC polarized proton operation are 28.6975 and 29.6838, respectively, horizontal and vertical beta-beat are dominated by the 57th to 60th harmonics, which cannot be accurately sampled by only 160 BPMs available at RHIC. Hence, the stop band compensation method is not effective.

The segment-by-segment technique (SBST) first introduced at the Large Hadron Collider (LHC) for identifying large local gradient errors in interaction regions [14] was also successfully applied to RHIC [15]. This sets a favorable foundation of the development of global optics correction schemes.

Because of the powering scheme at RHIC [16], only the triplets and trim quadrupoles in interaction regions have independent power supplies. To fully utilize these available quadrupole correctors for global beta-beat correction, a correction scheme of using the beta-beat response matrix method was systematically studied. To further reduce betabeat in the arcs in the presence of lacking independently powered quadrupoles in the region, a new scheme of using a horizontal closed orbit bump at sextupoles in the arcs was explored. These correction schemes were successfully demonstrated during the 2013 RHIC polarized proton operation. The experimental results are reported in this paper.

A brief introduction of the ICA technique for RHIC optics measurement is presented in Sec. II. Section III presents the algorithm and experimental results of a global correction scheme using the beta-beat response matrix method. Experimental results of using a horizontal closed orbit bump at sextupoles for arc beta-beat correction are presented in Sec. IV.

\section{ICA FOR AC DIPOLE BASED OPTICS MEASUREMENT}

The model of ICA assumes that in a complex dynamical system an $m$-dimensional observable vector $\mathbf{X}(t)$ is related to an $n$-dimensional source signal vector $\mathbf{s}(t)$ as

$$
\mathbf{X}(t)=\mathbf{A s}(t)+\mathbf{N}(t),
$$

where the $m \times n(m \geq n)$ matrix $\mathbf{A}$ is called mixing matrix and $\mathbf{N}(t)$ is the noise vector which is assumed to be stationary, temporally white and statistically independent of source signal $\mathbf{s}(t)$. The goal of ICA is to determine the mixing matrix $\mathbf{A}$ and the source signals $\mathbf{s}(t)$ from the sampled observable vector $\mathbf{X}(t)$ without a priori knowledge of the underlying physical process. The source signals $\mathbf{s}_{i}$ are assumed to be mutually independent and temporally uncorrelated. The time-lagged covariance matrix $\mathbf{C}_{\mathbf{s}}(\tau)=\left\langle\mathbf{s}(t) \mathbf{s}(t+\tau)^{T}\right\rangle$ is therefore diagonal [6], i.e., $\left\langle\mathbf{s}_{i}(t) \mathbf{s}_{j}(t+\tau)^{T}\right\rangle=S_{i}(\tau) \delta_{i, j}$, where the superscript $T$ means a transpose, $\tau$ is a time-lag constant, and the average is taken over time. From Eq. (1) the relationship between the time-lagged covariance matrix $\mathbf{C}_{\mathbf{X}}(\tau)=\left\langle\mathbf{X}(t) \mathbf{X}(t+\tau)^{T}\right\rangle$ and $\mathbf{C}_{\mathbf{s}}(\tau)$ is obtained:

$$
\begin{gathered}
\mathbf{C}_{\mathbf{X}}(0)=\mathbf{A C}_{\mathbf{s}}(0) \mathbf{A}^{T}+\sigma^{2} \mathbf{I}, \\
\mathbf{C}_{\mathbf{X}}(\tau)=\mathbf{A C}_{\mathbf{s}}(\tau) \mathbf{A}^{T}, \quad \tau \neq 0,
\end{gathered}
$$

where the random noises are assumed to have identical distributions with standard deviation $\sigma$. Since $\mathbf{C}_{\mathbf{s}}(\tau)$ is diagonal, the mixing matrix $\mathbf{A}$ is found as the joint diagonalizer of $\mathbf{C}_{\mathbf{X}}(\tau)$. In the following, the algorithms of ICA to extract the mixing matrix $\mathbf{A}$ and source signals $\mathbf{s}$ are discussed.

First, a whitening procedure is applied to preprocess the raw data. The zero time-lagged covariance matrix $\mathbf{C}_{\mathbf{X}}(0)$ is decomposed by singular valued decomposition (SVD):

$$
\mathbf{C}_{\mathbf{X}}(0)=\left(\mathbf{U}_{1}, \mathbf{U}_{2}\right)\left(\begin{array}{cc}
\boldsymbol{\Lambda}_{1} & 0 \\
0 & \boldsymbol{\Lambda}_{2}
\end{array}\right)\left(\begin{array}{c}
\mathbf{U}_{1}^{T} \\
\mathbf{U}_{2}^{T}
\end{array}\right),
$$

where $\mathbf{U}_{1}$ and $\mathbf{U}_{2}$ are orthogonal matrices, $\boldsymbol{\Lambda}_{1}$ and $\boldsymbol{\Lambda}_{2}$ are diagonal matrices with $\min \left(\operatorname{diag}\left[\boldsymbol{\Lambda}_{1}\right]\right) \geq$ $\lambda_{c}>\max \left(\operatorname{diag}\left[\boldsymbol{\Lambda}_{2}\right]\right) \geq 0, \lambda_{c}$ is a threshold to remove the singularity of the covariance matrix, and the elements of the $l \times l(l \leq n)$ diagonal matrix $\Lambda_{1}$ are arranged in a descending manner $\lambda_{1} \geq \lambda_{2} \geq \cdots \geq \lambda_{l}$. Using the matrix

$$
\mathbf{V} \equiv \boldsymbol{\Lambda}_{1}^{-1} \mathbf{U}_{1}^{T}
$$

an $l$-dimensional vector $\mathbf{Z}=\mathbf{V X}$ is constructed. $\mathbf{Z}$ is spatially white because it satisfies $\left\langle\mathbf{Z} \mathbf{Z}^{T}\right\rangle=\mathbf{I}$, where $\mathbf{I}$ is the $l \times l$ identity matrix. The whitening procedure removes redundant information and noise from the raw data and decorrelates and normalizes the data to facilitate the next step.

Using the whitened data $\mathbf{Z}$ and a set of time-lag constants $\left\{\tau_{k}\right\}(k=1,2, \ldots, K)$, the time-lagged covariance matrices are computed as $\left\{\mathbf{C}_{\mathbf{Z}}\left(\tau_{k}\right)=\left\langle\mathbf{Z}(t) \mathbf{Z}\left(t+\tau_{k}\right)\right\rangle\right\}$. The symmetrized time-lagged covariance matrices are formed as $\overline{\mathbf{C}}_{\mathbf{Z}}\left(\tau_{k}\right)=\left[\mathbf{C}_{\mathbf{Z}}\left(\tau_{k}\right)+\mathbf{C}_{\mathbf{Z}}^{T}\left(\tau_{k}\right)\right] / 2$ such that they are real and symmetric and thus their eigenvalue decompositions are well defined. At last, a Jacobi-like algorithm [17] is applied to find a unitary matrix $\mathbf{W}$ to joint diagonalize $\overline{\mathbf{C}}_{\mathbf{Z}}\left(\tau_{k}\right)$ such that $\overline{\mathbf{C}}_{\mathbf{Z}}\left(\tau_{k}\right)=\mathbf{W D}_{k} \mathbf{W}^{T}$, where $\mathbf{D}_{k}$ 's are diagonal matrices. The source signals are given by $\mathbf{s}=\mathbf{W}^{T} \mathbf{V X}$ and the mixing matrix by $\mathbf{A}=\mathbf{V}^{-1} \mathbf{W}$.

The turn by turn BPM data is normally composed of beam motions driven by different physical sources with their own characteristic frequencies, such as betatron 
oscillation, synchrotron oscillation, and electronic noises. A physical decomposition of the BPM data into these individual beam motions is of great interest for deeper understanding of beam dynamics. The source signals separated by ICA have nonoverlapping power spectra because of their properties of mutual independence. Therefore ICA is particularly suitable for the task of physical decomposition of BPM data. To apply ICA, we organize the data matrix as

$$
\mathbf{X}=\left(\begin{array}{cccc}
x_{1}(1) & x_{1}(2) & \ldots & x_{1}(N) \\
x_{2}(1) & x_{2}(2) & \ldots & x_{2}(N) \\
\vdots & \vdots & \ddots & \vdots \\
x_{M}(1) & x_{M}(2) & \ldots & x_{M}(N)
\end{array}\right)
$$

where $x_{i}(j)$ is the output of the $i$ th BPM at the $j$ th turn. The ICA algorithm is used to extract the source signal $\mathbf{s}$ and mixing matrix $\mathbf{A}$ in Eq. (1). $\mathbf{s}$ and $\mathbf{A}$ are also known as temporal function and spatial function, respectively. The combination of the $i$ th row of $\mathbf{s}, \mathbf{s}_{i}$, and the $i$ th column of $\mathbf{A}$, $\mathbf{A}_{i}$ is defined as the $i$ th mode. The correspondence of the $i$ th mode to a certain beam motion is identified by comparing the frequency of the temporal function $\mathbf{s}_{i}$ to the characteristic frequency of the beam motion. The spatial function $\mathbf{A}_{i}$ will then provide important information of beam motion. For example, the free betatron oscillation component $\mathbf{x}_{f}(t)$ of BPM data involves two modes with identical frequency spectra,

$$
\mathbf{x}_{f}(t)=\mathbf{A}_{f 1} s_{f 1}(t)+\mathbf{A}_{f 2} s_{f 2}(t),
$$

where $\mathbf{A}_{f 1}$ and $\mathbf{A}_{f 2}$ are the spatial functions. $s_{f 1}(t)$ and $s_{f 2}(t)$ are the cosinelike and sinelike source signals, respectively. The fast Fourier transform of these source signals reveals the betatron tune $\nu_{f}$. Using the spatial functions $\mathbf{A}_{f 1}$ and $\mathbf{A}_{f 2}$, the betatron amplitude function $\beta_{f, i}$ and phase advance $\psi_{f, i}$ at the $i$ th BPM can be extracted as [7]

$$
\begin{gathered}
\beta_{f, i}=\mathcal{F}\left(A_{f 1, i}^{2}+A_{f 2, i}^{2}\right), \\
\psi_{f, i}=\arctan \left(\frac{A_{f 2, i}}{A_{f 1, i}}\right),
\end{gathered}
$$

where $\mathcal{F}$ is a constant depending on initial conditions.

In the presence of the driven oscillation excited by an ac dipole, the measured optics are modified. The modified betatron amplitude function $\beta_{d, i}$ and phase advance $\psi_{d, i}$ observed at the $i$ th BPM are related to the optical functions in free betatron oscillation as [10]

$$
\begin{gathered}
\beta_{d, i}=\frac{1+\lambda^{2}-2 \lambda \cos \left[2\left(\psi_{f, i}-\pi \nu\right)\right]}{1-\lambda^{2}} \beta_{f, i}, \\
\tan \left(\psi_{d, i}-\pi \nu_{d}\right)=\frac{1+\lambda}{1-\lambda} \tan \left(\psi_{f, i}-\pi \nu_{f}\right),
\end{gathered}
$$

where $\lambda=\sin \left[\pi\left(\nu_{d}-\nu_{f}\right)\right] / \sin \left[\pi\left(\nu_{d}+\nu_{f}\right)\right], \nu_{d}$ is the driving tune of the ac dipole, and $\nu_{f}$ is the betatron tune of free oscillation. In Eq. (11), the reference point for phase advance is chosen to be the location of the ac dipole. Equations (10) and (11) show there is a systematic error in the measured optics. With a distance between the driven and the betatron tunes $\Delta \nu=\left|\nu_{d}-\nu_{f}\right|=0.01$, maximum systematic error on beta-beat and relative phase-beat between the $i$ th and $j$ th BPMs is approximately $7.0 \%$ and $5.5 \times 10^{-3} \mathrm{rad}$. Here relative phase-beat is defined as $\Delta \psi_{i j}=\left(\psi_{i, \text { measured }}-\psi_{j \text {, measured }}\right)-\left(\psi_{i \text {, model }}-\psi_{j \text {,model }}\right)$. The maximum systematic error of beta-beat and relative phase-beat can be reduced to $0.3 \%$ and $1.0 \times 10^{-3} \mathrm{rad}$, respectively, with the same operational conditions by averaging the optical functions from two measurements in which $\Delta \nu$ is of opposite signs. These residual systematic errors from the ac dipole can be negligible in comparison to the systematic error due to realistic BPM calibration error and noise. The systematic error of beta-beat from RHIC BPM calibration error is estimated to be approximately $2.0 \%$. RHIC BPM noise also introduces $2.0 \%$ systematic error on beta-beat. However, the relative phase-beat is immune to BPM calibration error and the contribution of BPM noise to relative phase-beat systematic error is below $0.01 \mathrm{rad}$. Details on the systematic error of optics measurement due to BPM errors are reported in the Appendix at the end of this paper.

Figure 1 shows the beta-beat for the $255 \mathrm{GeV}$ polarized proton beams in the Blue and Yellow rings during RHIC operation in 2013. In the horizontal plane, beta-beat is distributed smoothly along the Blue and Yellow rings. In both rings, the horizontal peak beta-beat is approximately $15 \%$. In the vertical plane, the peak beta-beat reaches $30 \%$ in the Blue ring and $60 \%$ in the Yellow ring. Hence, global beta-beat correction was explored.

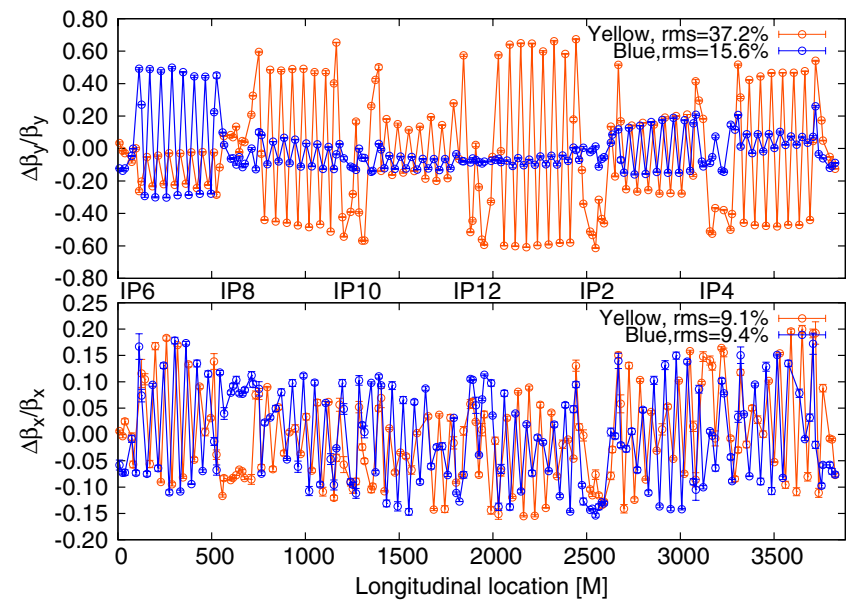

FIG. 1. Measured beta-beat with error bars in the horizontal (bottom) and vertical (top) plane for both rings at RHIC. A peak beta-beat of approximately $15 \%$ is observed in the horizontal plane for both rings. A $30 \%$ vertical peak beta-beat is found in the Blue ring and $60 \%$ in the Yellow ring. 


\section{BETA-BEAT RESPONSE MATRIX CORRECTION METHOD}

The beta-beat of betatron motion obeys a linear second order differential equation [11]. The beta-beat response matrix $\mathbf{R}$ with respect to the change of integrated strength $\Delta(K 1 L)$ of the quadrupoles is defined as

$$
\mathbf{B}=\mathbf{R} \mathbf{K},
$$

where $\mathbf{B}^{T}=\left(\frac{\Delta \vec{\beta}_{x}}{\beta_{x}}, \frac{\Delta \vec{\beta}_{y}}{\beta_{y}}, \Delta \nu_{x}, \Delta \nu_{y}\right)$ is composed of the beta-beat vectors and tune variations, and $\mathbf{K}^{T}=$ $\left(\Delta K 1 L_{1}, \Delta K 1 L_{2}, \ldots, \Delta K 1 L_{N}\right)$ represents the change of integrated strength of $N$ quadrupoles. In this case, $N$ quadrupoles are used to correct beta-beat measured from $M$ BPMs. In the presence of a limited number of quadrupole correctors, i.e., $M \gg N$, Eq. (12) describes an overdetermined system in which beta-beat is minimized. In practice, uncalibrated BPMs should be excluded from the measurement. Beta-beat measured from noisy BPMs should be assigned low weights. The tune variations must receive high weight in order to avoid large tune shifts which can cause excessive beam loss as well as polarization loss. Hence, different weighting factors are applied to Eq. (12) as

$$
\mathbf{W B}=\mathbf{W R K},
$$

where

$$
\mathbf{W}=\left(\begin{array}{cccc}
w_{1} & 0 & \ldots & 0 \\
0 & w_{2} & \ldots & 0 \\
\vdots & \vdots & \ddots & \vdots \\
0 & 0 & \ldots & w_{M}
\end{array}\right)
$$

Each diagonal element $w_{i}$ is the weighting factor for different BPMs and variations of tunes. The required correction strengths are computed by inverting the weighted response matrix WR to solve Eq. (13). Singular value decomposition (SVD) can be applied to decompose WR as

$$
\mathbf{W R}=\mathbf{U} \boldsymbol{\Gamma} \mathbf{V}^{T},
$$

where $\mathbf{U}$ is a real orthogonal $M \times M$ matrix, and $\mathbf{V}^{T}$ is a real orthogonal $N \times N$ matrix. $\boldsymbol{\Gamma}$ is a real diagonal $M \times N$ matrix with singular values $\boldsymbol{\Gamma}_{11}=\gamma_{1} \geq \boldsymbol{\Gamma}_{22}=$ $\gamma_{2} \geq \cdots \geq 0$. To remove singularities, all $\gamma_{i} \leq \gamma_{c}$ $(i>r)$ are set to $\gamma_{i}=0(i>r)$, where $\gamma_{c}$ is called tolerance level and $r$ is called the rank of WR. Once the SVD of WR is obtained, the generalized inverse $(\mathbf{W R})^{\dagger}$ is given by

$$
(\mathbf{W R})^{\dagger}=\mathbf{V} \boldsymbol{\Gamma}^{-1} \mathbf{U}^{T},
$$

where $\boldsymbol{\Gamma}^{-1}$ is a diagonal matrix with $\boldsymbol{\Gamma}_{11}^{-1}=1 /$ $\gamma_{1}, \ldots, \boldsymbol{\Gamma}_{r r}^{-1}=1 / \gamma_{r}$ and 0 for all diagonal elements with $i>r$. The required correction strengths for global beta-beat correction $\mathbf{K}_{\text {cor }}$ are computed as

$$
\mathbf{K}_{\mathrm{cor}}=-(\mathbf{W R})^{\dagger} \mathbf{W B}
$$

The solution given in Eq. (17) is equivalent to a weighted $\chi^{2}$ minimization of the beta-beat. In the event of large betabeat, multiple iterations of correction based on Eq. (17) are necessary.

According to the method discussed above, systematic computer simulations were carried out to find the optimum correction. A total of 72 triplets and trim quadrupoles in the interaction regions with independent power supplies are used as beta-beat correctors, while two families of arc quadrupoles are also included as tune correctors. Table I summarizes parameters for beta-beat corrections in both rings. Since the horizontal and vertical ac dipole are common to both rings, the betatron tunes of both rings were adjusted to be the same in the corresponding plane for simultaneous optics measurement.

The response matrix $\mathbf{R}$ was numerically computed from the design model by individually perturbing the integrated strength of each quadrupole and recording the unit response of beta-beat and tunes. The correction is computed with a careful choice of weighting factors and rank of response matrix $\mathbf{R}$ such that the global beta-beat is reduced as much as possible with minimum tune variations.

Figure 2 shows the computed relative correction strengths $\Delta(K 1 L) / K 1 L$ for the Blue ring. All of the relative corrections are within $1.0 \%$. The relative changes in many trim quadrupoles are large because trim quadrupoles are normally set at a low field. Figure 3 shows a simulation of the evolution of tunes and rms beta-beat in the Blue ring along a ramp-up process of the computed correction. There are no tune variations after $100 \%$ correction strength. During the ramp-up process, the excursions of tune changes are within $5 \times 10^{-4}$. The rms beta-beat in both planes is reduced smoothly during the correction ramp-up process. The small increase of horizontal rms beta-beat at the end of the correction ramp-up process is because of decreasing vertical beta-beat and tune compensation. The measured rms beta-beat in Fig. 3 shows a good agreement with the predicted values.

Figure 4 shows the measured beta-beat with and without correction. The horizontal peak beta-beat was successfully reduced from $15 \%$ to $8 \%$. In the vertical plane, significant suppression of beta-beat was also achieved in the arc between IP6 and IP8 as well as the one between IP2 and

TABLE I. Parameters for optics measurement and correction.

\begin{tabular}{lcc}
\hline \hline Parameter & Blue & Yellow \\
\hline Horizontal tune $\nu_{x}$ & 28.6975 & 28.6975 \\
Vertical tune $\nu_{y}$ & 29.6838 & 29.6838 \\
Number of horizontal BPM (used/total) & $157 / 160$ & $156 / 160$ \\
Number of vertical BPM (used/total) & $158 / 160$ & $158 / 160$ \\
Number of beta-beat correctors & 72 & 72 \\
Number of tune corrector families & 2 & 2 \\
\hline \hline
\end{tabular}




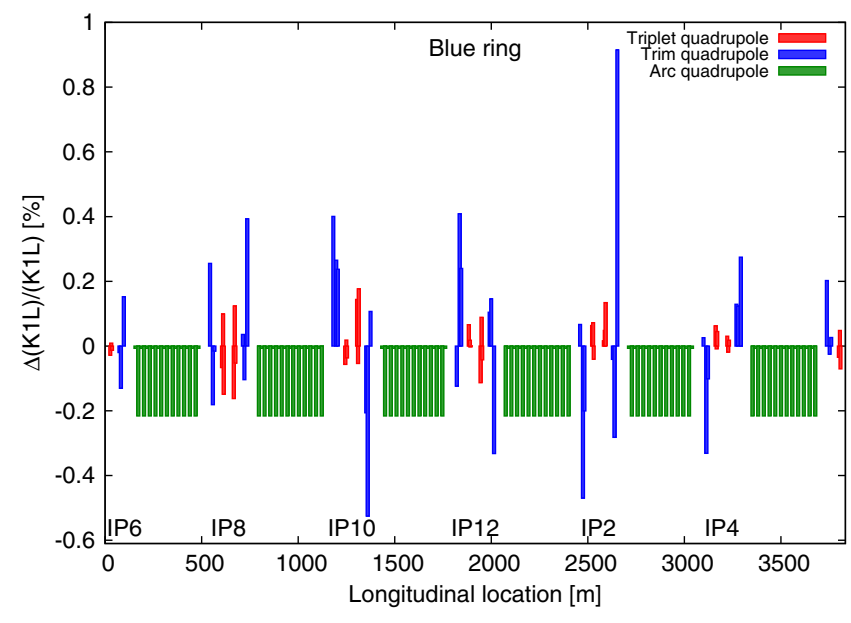

FIG. 2. Relative changes of quadrupole integrated strength as a function of quadrupole locations in the Blue ring.

IP4. The vertical peak beta-beat was reduced from $40 \%$ to $14 \%$.

Now we discuss the Yellow ring which has excessively large beta-beat in the vertical plane. The relative correction strengths for the first trial of corrections are shown in Fig. 5 by the hollow bars. All relative correction strengths are within $0.6 \%$. The large relative correction strengths in the arc quadrupoles were due to compensation of the large tune shifts caused by the triplet and trim quadrupoles employed to minimize the beta-beat. Figure 6 shows the simulated evolution of tunes and rms beta-beat along the ramp-up process of the first correction as well as the measured rms beta-beat at $100 \%$ correction strength. The excursions of tune variations are within $2 \times 10^{-3}$. The rms beta-beat is reduced smoothly as correction strength increases. At full correction strength, the 5\% measured horizontal rms betabeat is lower than the predicted $5.4 \%$ value. The top plots
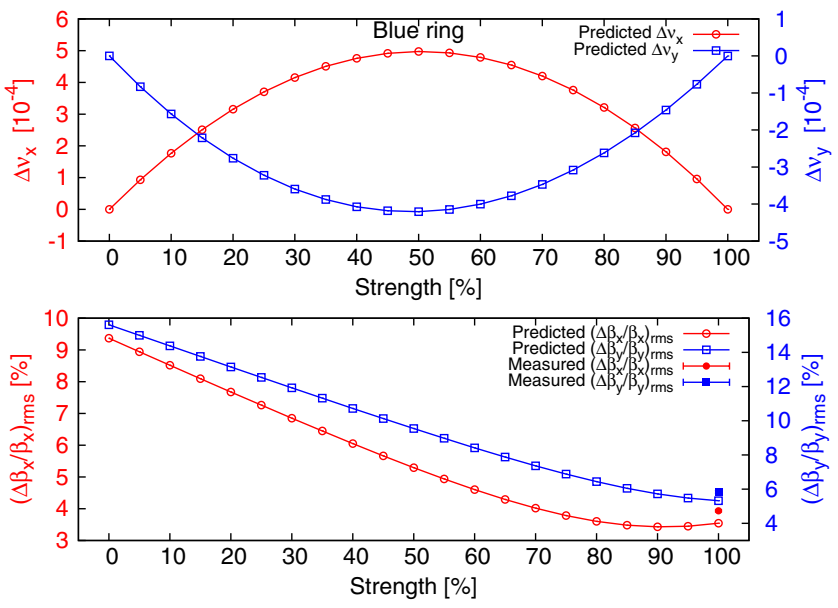

FIG. 3. Simulated evolution of tunes and rms beta-beat along a ramp-up process of the computed correction for the Blue ring. The measured rms beta-beat with error bars at $100 \%$ correction strength is also shown.

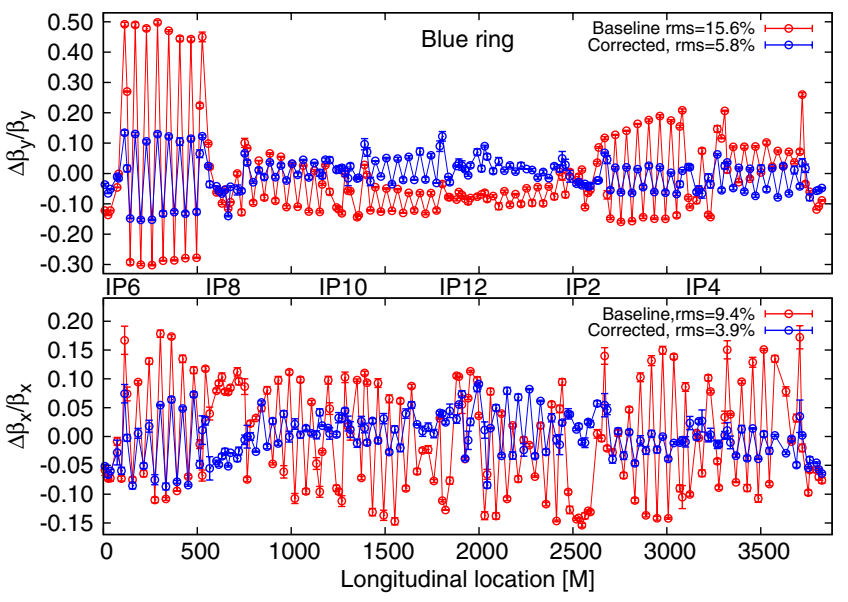

FIG. 4. Baseline and corrected horizontal (bottom) and vertical (top) beta-beat with error bars for the Blue ring. Horizontal peak beta-beat was corrected from $15 \%$ to $8 \%$, while vertical peak beta-beat from $40 \%$ to $14 \%$.

in Figs. 7 and 8 show the measured beta-beat of the Yellow ring with and without the correction. The horizontal peak beta-beat was reduced to $12 \%$. However, in the vertical plane there was still a peak beta-beat as large as $20 \%$, and the $11 \%$ measured vertical rms beta-beat is about 2 times of the prediction shown in Fig. 6. This is due to the initial large beta-beat in the vertical plane. Hence, a second iteration was exercised.

The results of the second iteration of correction along with the first iteration are shown in the two bottom plots in Figs. 7 and 8. After the second iteration, significant vertical beta-beat reduction was achieved, and the peak beta-beat was successfully reduced to approximately $10 \%$ for both planes. The computed relative correction strengths are shown in Fig. 5 by the solid bars. The relative correction

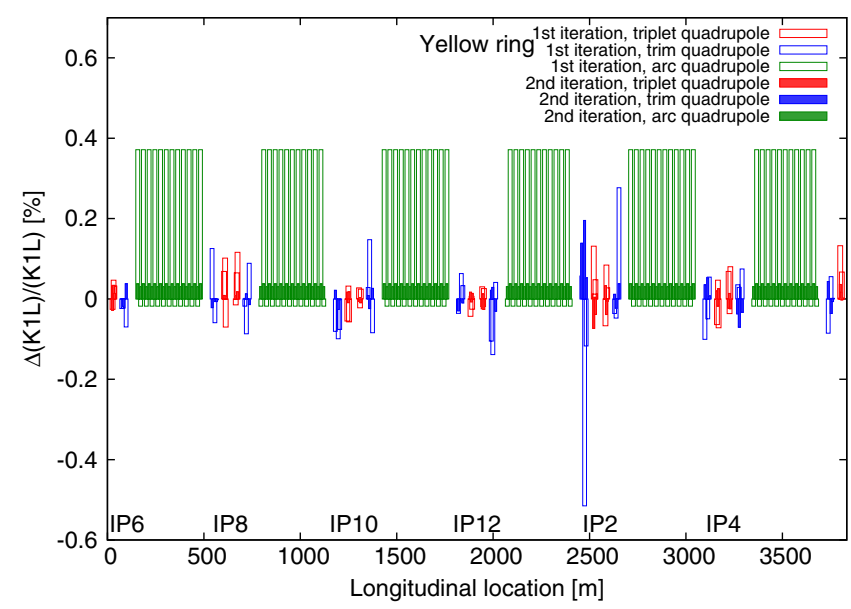

FIG. 5. Relative changes of quadrupole integrated strength as a function of quadrupole locations in the Yellow ring for the first iteration (hollow bars) and second iteration (solid bars), respectively. 

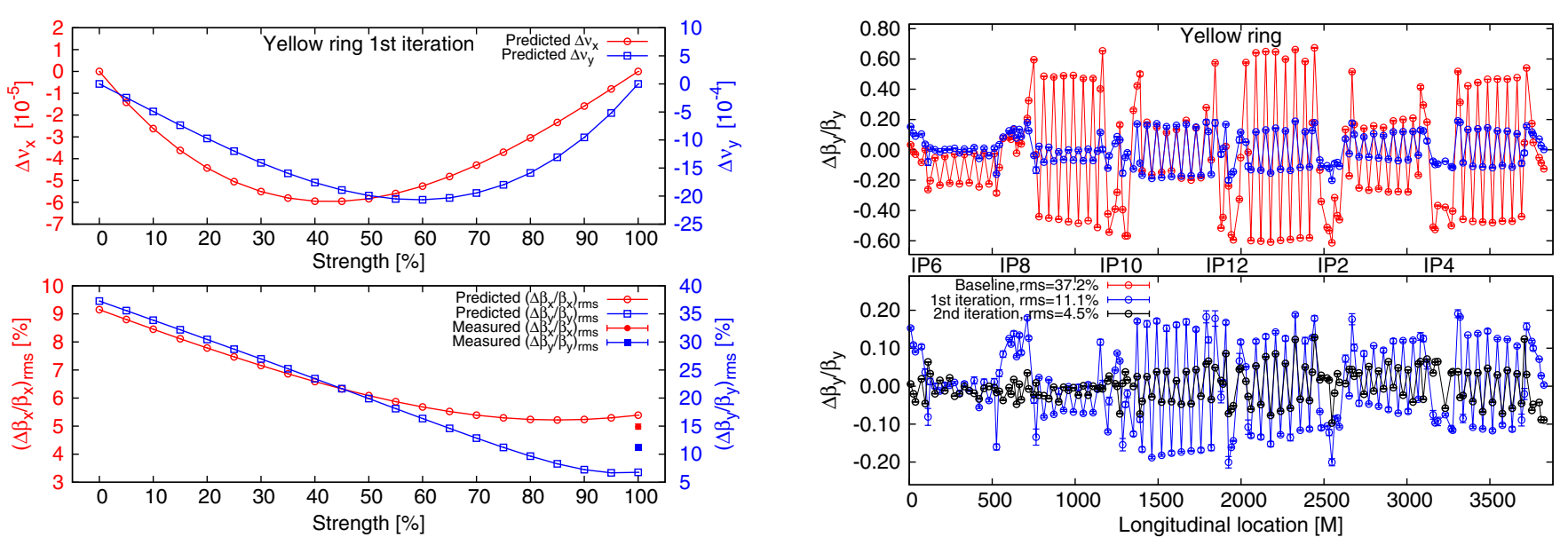

FIG. 6. Simulated evolution of tunes and rms beta-beat along a ramp-up process of the computed first iteration of corrections for the Yellow ring. The measured rms beta-beat with error bars at $100 \%$ of correction strength is also shown.

strengths are smaller than those for the first iteration. Similar to Fig. 6, the evolution of tunes and rms betabeat for the second iteration was also computed and shown in Fig. 9. The excursions of tune variations are within $4 \times 10^{-4}$ and the changes of rms beta-beat are smooth. At full correction strength, the $4 \%$ measured horizontal rms beta-beat matches the predicted value of $3.8 \%$, and the deviation between measured and predicted vertical rms beta-beat is $0.6 \%$ after the second iteration.

The phase difference between two BPMs $\psi_{i j}$, another measure of linear optics, is highly correlated with betabeat. It is expected for a successful optics correction scheme that the minimization of beta-beat should also minimize the relative phase-beat $\Delta \psi_{i j}$. Thanks to the high quality BPMs at RHIC and on-going efforts in further

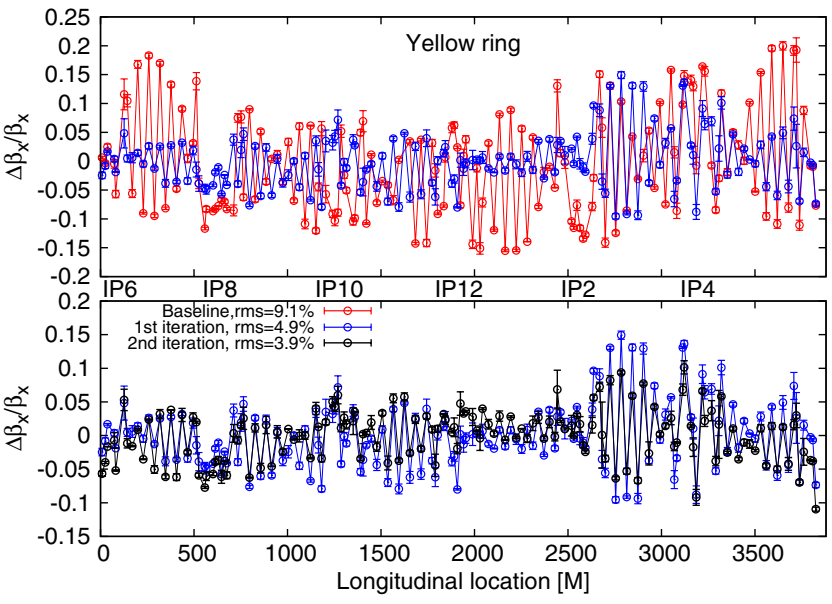

FIG. 7. Baseline and corrected horizontal beta-beat with error bars for the Yellow ring. The peak beta-beat was reduced from $15 \%$ to $12 \%$ after the first iteration of correction, and further reduced to $10 \%$ after the second iteration.
FIG. 8. Baseline and corrected vertical beta-beat with error bars for the Yellow ring. The peak beta-beat was reduced from $60 \%$ to $20 \%$ after the first iteration of correction, and further reduced to $10 \%$ after the second iteration.

improving BPM performance over a decade of RHIC operation, our optics correction based on beta-beat response matrix also results in a significant reduction in relative phase-beat between consecutive BPMs, especially in the vertical plane, which is shown in Fig. 10. This also validates our optics correction method.

Because of the difference between the real machine and the ideal model, a variation of the tunes on the order of $10^{-3}$ was observed after the corrections. This can be corrected afterward. In conclusion, our optics correction method based on beta-beat response matrix has successfully reduced both horizontal and vertical peak beta-beats to $10 \%$ in both rings. Good agreement was also found between measurements and predictions. There were no possibilities to apply additional iterations of correction in this experiment due to the limited beam time. For further
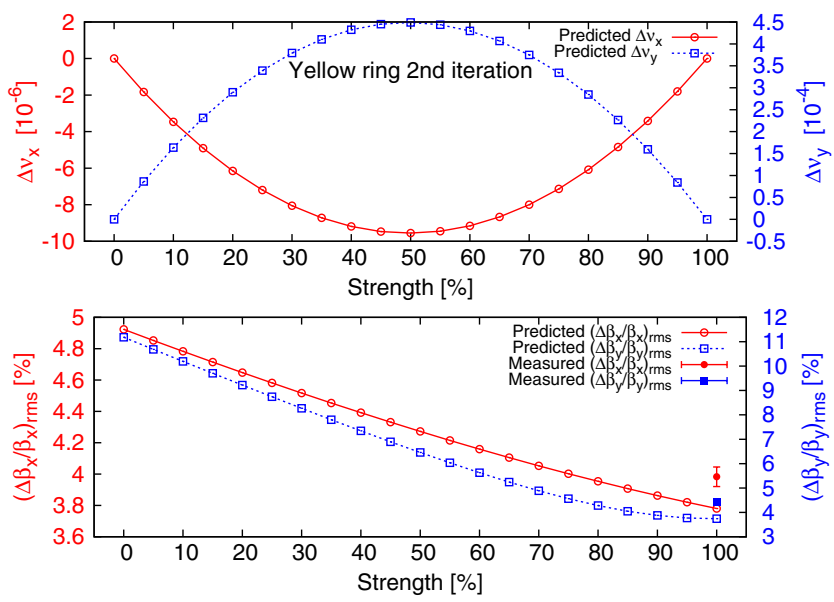

FIG. 9. Simulated evolution of tunes and rms beta-beat along a ramp-up process of the computed second iteration of corrections for the Yellow ring. The rms measured beta-beat with error bars at $100 \%$ of correction strength is also shown. 


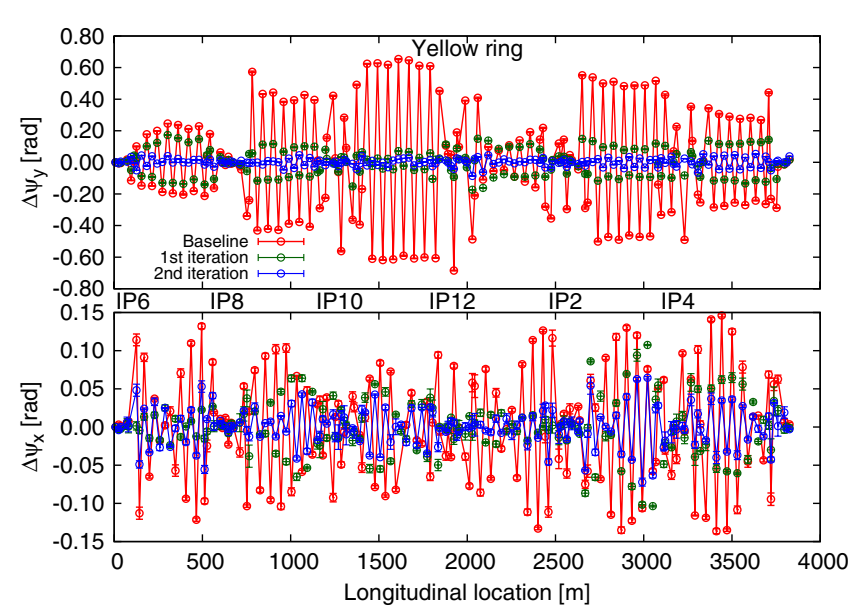

FIG. 10. Baseline and corrected horizontal (bottom) and vertical (top) relative phase-beat with error bars for the Yellow ring. The reduction of relative phase-beat after correction of beta-beat is evident.

correction of arc beta-beat, additional techniques are needed.

\section{ARC BETA-BEAT CORRECTION USING CLOSED ORBIT BUMP AND SEXTUPOLE}

In high energy accelerators, very often the number of available arc quadrupoles with independent power supplies is limited. In RHIC, all arc quadrupoles are grouped into two families and each family is powered by a common power supply [16]. Therefore gradient errors in the arcs cannot be effectively compensated with global correction using only quadrupoles in the interaction region. However, horizontal orbit correctors in the arcs with $\pi$ phase advance can be paired to generate closed orbit bumps at sextupoles and achieve a feed-down normal quadrupole field [11],

$$
\begin{aligned}
& B_{x}=B_{2} x_{\mathrm{co}} y, \\
& B_{y}=B_{2} x_{\mathrm{co}} x,
\end{aligned}
$$

where $B_{2}=\partial^{2} B_{y} / \partial x^{2}$ and $x_{\text {co }}$ is the horizontal closed orbit offset. This feed-down quadruple field can be used to correct arc beta-beat [18]. Once the response matrix of arc beta-beat to the amplitude of the closed orbit bump is obtained from the ideal model, an SVD inversion method discussed in Sec. III can be applied to compute the required pattern of closed orbit bumps. Although the principle is well known, yet it still needs to be demonstrated that this technique can be implemented in a high energy accelerator like RHIC, as well as LHC, where precise control of linear optics is required to facilitate optics manipulations. Such an example can be the achromatic telescopic squeezing (ATS) [19] for further increasing of luminosity at LHC.

A proof-of-principle experiment was carried out in the Yellow ring at beam energy of $255 \mathrm{GeV}$ during the RHIC polarized proton run in 2013. SBST was first applied to correct large local beta-beat. The beta-beat response matrix method using quadrupoles in interaction regions and arcs was applied to reduce peak beta-beat in both planes to approximately $10 \%$ to facilitate arc beta-beat correction. The computed horizontal closed orbit bumps were then applied by the closed orbit feedback system [20]. Figure 11 shows the computed horizontal closed orbit required for arc beta-beat correction. The corresponding measured horizontal closed orbit in Fig. 11 shows a good agreement with prediction in all arcs. At around $s=500 \mathrm{~m}$, the measured closed orbit missed a computed orbit bump. This is because in the computed closed orbit three horizontal orbit correctors were used to match the closed orbit at the beginning of the interaction region at IP8. But, in the experiment the orbit feedback system used more than three horizontal orbit correctors and resulted in smaller bumps at the same location. However, these orbit bumps do not affect the beta-beat correction because there are no sextupoles in this location. The large closed orbit bump measured at around $s=1200 \mathrm{~m}$ near IP10 is intrinsic to RHIC for beam dump. This bump is not considered in the computed closed orbit. However, it does not affect the correction results since there are no setupoles in this region as well. No beam loss was observed with this closed orbit pattern in which a maximum excursion about $8.5 \mathrm{~mm}$ was observed in between IP6 and IP8. The correction results are shown in Fig. 12. In the horizontal plane, beta-beat reduction was seen clearly in the arc between IP10 and IP12 as well as the arc between IP4 and IP6, while beta-beat in the other arcs remains about the same. Peak beta-beat was successfully reduced to approximately $7 \%$. In the vertical plane, significant beta-beat reduction was observed in the four arcs between IP10 and IP4. Especially in the arc between IP2 and IP4, peak beta-beat was remarkably reduced from $10 \%$ to $4 \%$. Overall, the vertical peak beta-beat was reduced from $10 \%$ to $7 \%$.

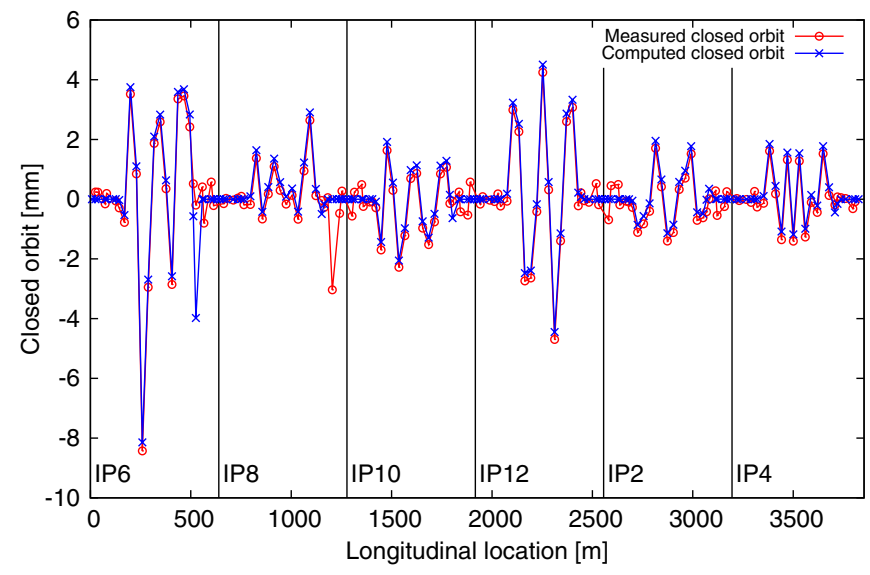

FIG. 11. Measured and computed horizontal closed orbit for correction of arc beta-beat in the Yellow ring. A maximum excursion of $8.5 \mathrm{~mm}$ was observed in the arc between IP6 and IP8. 


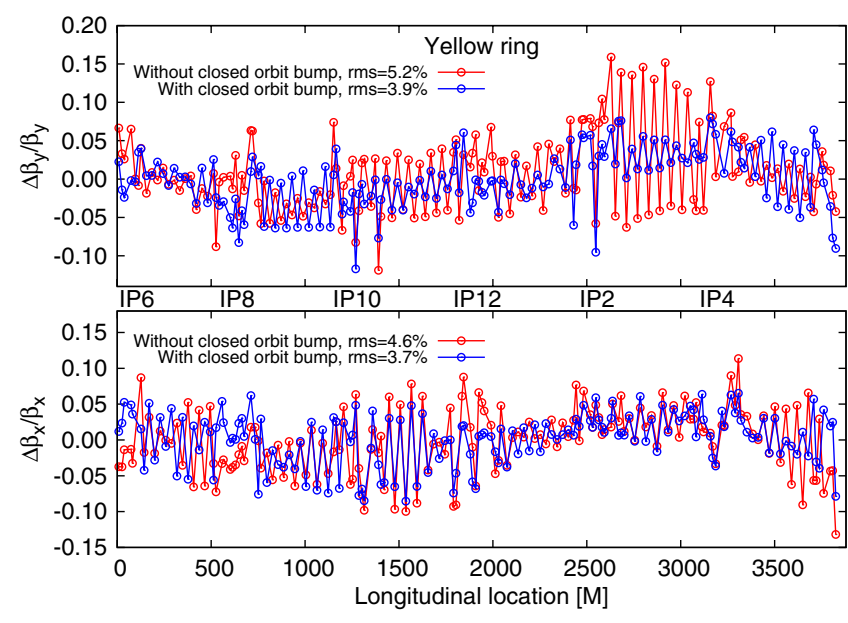

FIG. 12. Horizontal (bottom) and vertical (top) residual betabeat with and without the horizontal closed orbit bump displayed in Fig. 11 for the Yellow ring. The peak beta-beat was reduced to $7 \%$ for both planes with the closed orbit bump.

Because of limited beam time, only one set of measurements was taken such that no error bars are available for this beta-beat. However, experiences of previous measurements show that a typical average value of error bar is about $0.5 \%$. There were not any opportunities to further explore beta-beat correction using larger horizontal closed orbit bumps within limited beam time. Nonetheless, the successful demonstration of using horizontal closed orbit bump at sextupoles to correct arc beta-beat at RHIC polarized proton store energy shows this technique is feasible for high energy accelerator operations.

Figure 12 also shows a modulation of the measured betabeat such that the offset of beta-beat varies similarly as a sinusoidal wave with a period equal to the circumference of the ring. The amplitude of the variation is about $2 \%$. This modulation effect may have resulted from distributed coupling errors, such as triplet quadrupole roll errors and skew quadrupole errors. Further investigations are needed to identify the sources of this modulation effect.

\section{CONCLUSION AND DISCUSSION}

The technique of using ICA to accurately extract optical functions from turn by turn BPM data of ac dipole driven betatron oscillation was developed and by the first time applied to RHIC. With a limited number of quadrupole correctors, a global correction scheme using the beta-beat response matrix method was experimentally demonstrated to reduce the peak beta-beat to $10 \%$. Peak beta-beat was further reduced to $7 \%$ during a proof-of-principle experiment of arc beta-beat correction using horizontal closed orbit bumps at sextupoles.

ICA may also be applied to measure many other beam dynamical parameters more precisely, such as global linear coupling. The response matrix correction method can be improved by including global linear coupling as well as dispersion function corrections.

\section{ACKNOWLEDGMENTS}

The authors would like to thank S. Tepikian for his help in providing the RHIC design model. The authors would also like to thank the engineers in the beam instrumentation group as well as RHIC operators for their technical support. This work is supported in part by grants from the U.S. Department of Energy under Contract No. DE-FG0212ER41800, and the National Science Foundation NSF PHY-1205431.

\section{APPENDIX: ANALYSIS OF OPTICS MEASUREMENT SYSTEMATIC ERRORS DUE TO BPM CALIBRATION ERROR AND NOISE}

The systematic error in the linear optics measurements presented in this paper mainly comes from BPM calibration error as well as BPM noise. Numerical simulations were carried out to study optics measurement systematic error due to BPM calibration error and noise. For each simulation, a single on-momentum particle was driven by an ac dipole for 3072 turns. The ac dipole kick amplitude was linearly ramped up to its maximum value during the first 1024 turns. It was then kept constant for the second 1024 turns, and linearly ramped down during the last 1024 turns. The maximum ac dipole kick amplitude was chosen to produce a coherent oscillation amplitude about $300 \mu \mathrm{m}$ in the middle of an arc, a similar level of driven oscillation amplitude as observed during experiment. Optics was obtained by averaging the optics from simulated turn by turn data with an ac dipole driven tune set on either side of the betatron tune at a distance of 0.01.

Figure 13 shows the effect of BPM calibration error on the systematic error of beta-beat as well as phase-beat. For each data point in the plot, each BPM was assigned a

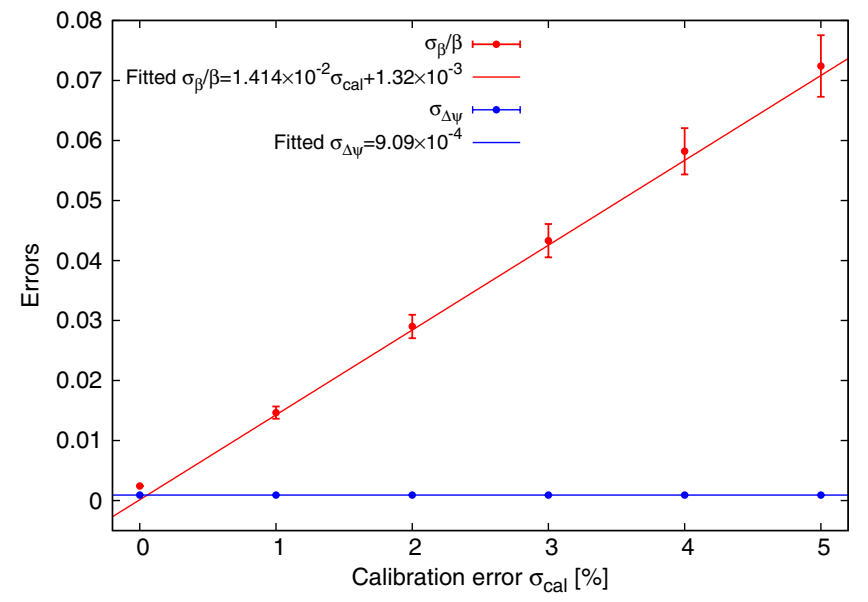

FIG. 13. Estimation of rms errors $\sigma_{\beta} / \beta$ and $\sigma_{\Delta \psi}$ with various BPM random calibration errors $\sigma_{\text {cal }}$. The estimation at each random calibration error $\sigma_{\text {cal }}$ is made by repeating the measurement of $\beta$ and $\Delta \psi 500$ times with random Gaussian calibration errors added to each BPM. The error bars reflect the standard deviations at corresponding noise levels. 


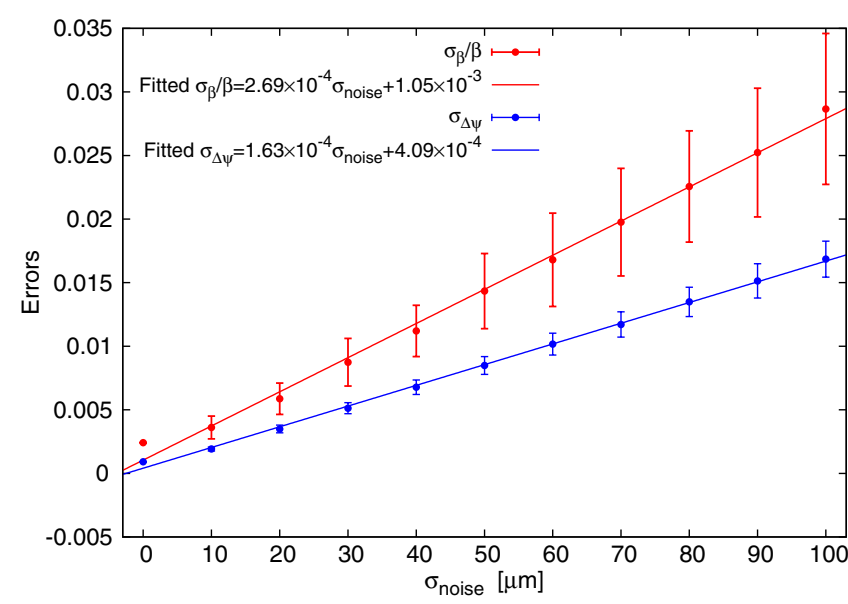

FIG. 14. Estimation of rms errors $\sigma_{\beta} / \beta$ and $\sigma_{\Delta \psi}$ with various random noise levels $\sigma_{\text {noise }}$. The estimation at each noise level $\sigma_{\text {noise }}$ is made by repeating the measurement of $\beta$ and $\Delta \psi 500$ times with white Gaussian random noises added to each BPM. The error bars reflect the standard deviations at corresponding noise levels.

random calibration error with Gaussian distribution. As shown in Fig. 13, $\sigma_{\beta} / \beta$ increases linearly with respect to $\sigma_{\text {cal }}$, while $\sigma_{\Delta \psi}$ is immune to BPM calibration error. At RHIC, BPM calibration error is estimated to be at the $1.0 \%$ level [21], which corresponds to a less than $2.0 \%$ systematic error on beta-beat based on the simulation result in Fig. 13.

The effect of BPM noise on the systematic error of betabeat as well as phase-beat is shown in Fig. 14. The results are consistent with the analytic estimation of PCA in Eq. (17) of Ref. [5]. RHIC BPM noise was then estimated by SVD analysis of all turn by turn BPM data during experiment [22]. Figure 15 shows the calculated histogram of noise level for all BPMs. Evidently, the maximum BPM

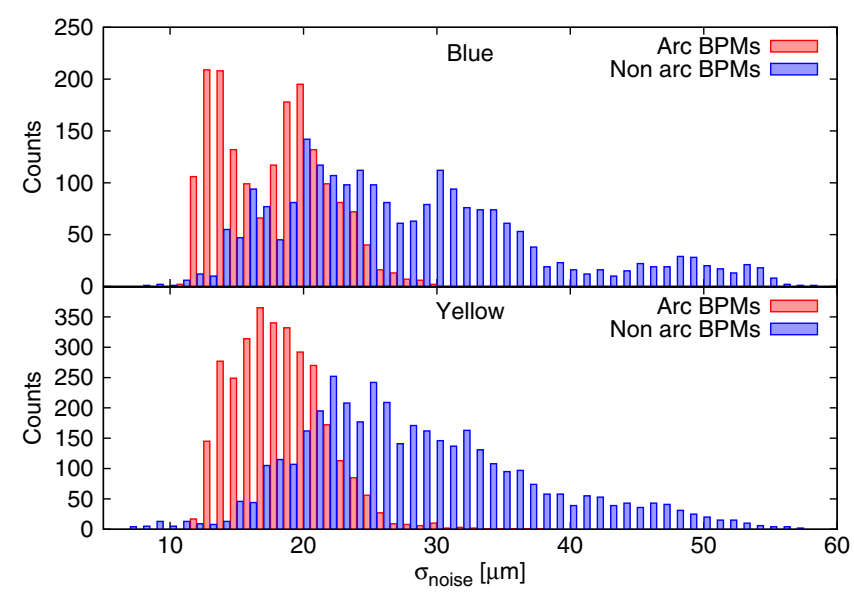

FIG. 15. Histogram of BPM noise levels estimated from a total of 37 turn by turn BPM data. In each set of data there are 68 arc BPMs and 88 other BPMs on average. noise level is below $60 \mu \mathrm{m}$, which contributes to less than $2 \%$ systematic error in beta-beat as shown in Fig. 14. Figure 15 also shows the arc BPMs are less noisy than the BPMs in the interaction regions.

[1] W. Fischer, Z. Altinbas, M. Anerella, E. Beebe, M. Blaskiewicz, D. Bruno, W. Dawson, D. Gassner, X. Gu, R. Gupta et al., in Proceedings of IPAC 2011, San Sebastián, Spain (EPS-AG, Spain, 2011); G. RobertDemolaize, A. Marusic, S. Tepikian, and S. White, in Proceedings of IPAC 2011, San Sebastin, Spain (EPS-AG, Spain, 2011); C. Montag, M. Bai, J. BeebeWang, W. Fischer, Y. Luo, N. Malitsky, T. Roser, T. Satogata, and S. Tepikian, in Proceedings of EPACO8, Genoa, Italy (EPS-AG, Genoa, Italy, 2008).

[2] S. Peggs, in Proceedings of the Particle Accelerator Conference, 1999 (IEEE, New York, 1999), Vol. 3, pp. 1572-1574.

[3] M. Bai, S. Y. Lee, J. W. Glenn, H. Huang, L. Ratner, T. Roser, M. J. Syphers, and W. van Asselt, Phys. Rev. E 56, 6002 (1997).

[4] Y. T. Yan, Y. Cai, F.-J. Decker, J. Irwin, J. Seeman, S. Ecklund, M. Sullivan, J. Turner, and U. Wienands, SLAC Report No. SLAC-PUB-10369, 2004.

[5] C. X. Wang, V. Sajaev, and C. Y. Yao, Phys. Rev. ST Accel. Beams 6, 104001 (2003).

[6] J.F. Cardoso, in Proceedings of IEEE International Conference on Acoustic, Speech, and Signal Processing, Seattle, WA (IEEE, Seattle, 1989), Vol. 4, pp. 1941-1944.

[7] X. Huang, S. Y. Lee, E. Prebys, and R. Tomlin, Phys. Rev. ST Accel. Beams 8, 064001 (2005).

[8] F. Wang, and S. Y. Lee, Phys. Rev. ST Accel. Beams 11, 050701 (2008).

[9] X. Pang and S. Y. Lee, J. Appl. Phys. 106, 074902 (2009).

[10] R. Miyamoto, S. E. Kopp, A. Jansson, and M. J. Syphers, Phys. Rev. ST Accel. Beams 11, 084002 (2008).

[11] S. Y. Lee, Accelerator Physics (World Scientific, Singapore, 2004).

[12] J. Safranek, Nucl. Instrum. Methods Phys. Res., Sect. A 388, 27 (1997).

[13] C. Gardner, BNL Collider-Accelerator Department AGS Technical Note No. 321, 1989.

[14] R. Tomás, T. Bach, R. Calaga, A. Langner, Y. Levinsen, E. Maclean, T. Persson, P. Skowronski, M. Strzelczyk, G. Vanbavinckhove et al., Phys. Rev. ST Accel. Beams 15, 091001 (2012).

[15] M. Bai, J. Aronson, M. Blaskiewicz, Y. Luo, G. RobertDemolaize, S. White, and G. Vanbavinckhove, in Proceedings of IPAC 2011, San Sebastián, Spain (Ref. [1]).

[16] RHIC configuration manual [http://www.c-ad.bnl.gov/ Accel/RCM_TOC.htm].

[17] J. F. Cardoso and A. Souloumiac, SIAM J. Matrix Anal. Appl. 17, 161 (1996).

[18] G. Yocky, in Proceedings of the 10th European Particle Accelerator Conferences, Edinburgh, Scotland (EPS-AG, Edinburgh, Scotland, 2006).

[19] S. Fartoukh, Report No. CERN-ATS-2011-161, 2011. 
[20] M. Minty, A. Marusic, A. Curcio, C. Dawson, C. Degen, W. Fischer, R. Hulsart, Y. Luo, G. Marr, K. Mernick et al., in Proceedings of IPAC 2011, San Sebastián, Spain (Ref. [1]).

[21] M. Bai, P. Cameron, P. Cerniglia, R. Connolly, J. Cupolo, C. Degen, A. Drees, R. Fliller, D. Gassner, J. Mead et al.,
Nucl. Instrum. Methods Phys. Res., Sect. A 499, 372 (2003).

[22] C.-x. Wang, M. Borland, V. Sajaev, and K.-J. Kim, in Proceedings of the 19th Particle Accelerator Conference, Chicago, Illinois, 2001 (IEEE, Piscataway, NJ, 2001), Vol. 2, pp. 1354-1356. 